\title{
Oscillatory Dynamical Switching System of Bulk Ferroelectrics
}

\author{
${ }^{1}$ M.N.A. Halif, ${ }^{2,3}$ S. Daud and ${ }^{4}$ Junaidah Osman \\ ${ }^{1}$ School of Microelectronic Engineering, Kolej Universiti Kejuruteraan Utara Malaysia (KUKUM), \\ Jalan Kangar-Arau, 02600 Jejawi, Perlis, Malaysia. \\ ${ }^{2}$ School of Computer and Communication Engineering, Kolej Universiti Kejuruteraan Utara Malaysia \\ (KUKUM), Jalan Kangar-Arau, 02600 Jejawi, Perlis, Malaysia. \\ ${ }^{3}$ Faculty Computer Science and Information System, Universiti Teknologi Malaysia, \\ 81310 UTM, Skudai, Johor, Malaysia \\ ${ }^{4}$ School of Physics, Universiti Sains Malaysia, 11800 USM, Penang, Malaysia
}

\begin{abstract}
This study gives a detailed account of calculation of the bulk ferroelectric (FE) oscillatory dynamical system switching to first and second-order phase transition, respectively. All the formalism is delineated in the framework of Landau free-energy expansion and Landau-Khalatnikov (LK) equation of motion where the effect of external energy may affray FE atoms similar to the spring damped oscillatory system. Here we scrutinized the switching properties from free-energy expansion and hysteresis loop. The polarization and current switching appropriate to the estimated complete switching time, changing of temperature, electric field and damping are scrutinized and discussed.
\end{abstract}

Key words: Phase Transitions, Coercive Field, Polarization Switching

\section{INTRODUCTION}

Ferroelectric (FE) materials are of special interest to developers of the next generation of such devices because they manifest polarized electronic states that can symbolize bits of information. Moreover, these materials preserve their polarization states without consuming electrical power, making FE the subject of intense study for non-volatile memory applications in which data is stored even when the power is turned off. FE materials are characterized by reversible spontaneous polarization $\mathrm{p}_{\mathrm{s}}$. The spontaneous polarization $\mathrm{p}_{\mathrm{s}}$ arise due to non-centrosymmetric set in order of ion unit cells which produce a permanent electric dipole moment affiliated with the unit cell. $\mathrm{p}_{\mathrm{s}}$ in FE can be reversed by applying the external electric field $\mathrm{e}$ where the arrangement is $\mathrm{e}>\mathrm{e}_{\mathrm{c}}$ (the coercive field). This reversal phenomenon is called switching. Lines and Glass ${ }^{[1]}$ state that, $p_{s}$ eventuated in FE phase correlates with the Curie temperature $T_{c}$ where $p_{s} \neq 0$ if $\mathrm{T}<\mathrm{T}_{\mathrm{c}}$ and $\mathrm{p}_{\mathrm{s}}=0$ if $\mathrm{T}>\mathrm{T}_{\mathrm{c}}$, the FE phase and paraelectric (PE) phase, respectively. In the first-order ferroelectric-paraelectric phase transitions, $p_{s}$ may have an essential value at temperatures approaching to $T_{c}$, while for second-order, the decrease of $p_{s}$ as temperature $T \sim T_{c}$ is more continuous.

Ricinschi et al..$^{[2]}$ and Tan et al. ${ }^{[3]}$ Give details of switching behavior of bulk, semi-infinite and thin film
FE in second and first-order phase transition respectively where both exchange options in relaxational switching system. As we know, in practice, the atoms in FE materials may fluctuate oscillatory due to the influence of external energy. As far as we know, there is no basic discussion to estimate the switching time of the FE bulk materials in terms of oscillatory dynamical system. It may be used as a preliminary step towards corresponding calculations for the symmetric and asymmetric FE thin film. Currently, there are many experimental observations to study the switching behavior of FE materials; for example, Merz ${ }^{[4]}$ using optical methods to observe the $\mathrm{BaTiO}_{3}$ domain structure, recorded the motion of $90^{\circ}$ and $180^{\circ}$ domain walls in single crystal $\mathrm{BaTiO}_{3}$ thin film using TEM and Shur et $a l .{ }^{[5]}$ Found that the domain dynamics essentially depend on the applied electric field.

From the theoretical consideration in this study, we focused our study to represent the estimate time of bulk FE for both cases: first and second-order phase transition. From previous results ${ }^{[6]}$, we estimated that the switching time for second-order case verging on $\mathrm{t} \sim 1.064 \times 10^{-13} \mathrm{~s}$. Although this value may represent very fast switching, since in practice the value is in nanosecond but it is authentic because the effect of domain wall movement and geometry effect e.g. films are neglected. On the other hand, the switching study of polarization in bulk FE gives us the clear view of what

Corresponding Author: M.N.A. Halif, School of Microelectronic Engineering, Kolej Universiti Kejuruteraan Utara Malaysia

Jalan Kangar-Arau, 02600 Jejawi, Perlis, Malaysia

E-mail: m.nazri@kukum.edu.my Tel: +60-4-9798301 Fax: +60-4-9798305

1073 
may happen in bulk ferroelectric and what are the fastest times of FE switching without any causal effect. Larsen et al. ${ }^{[7,8]}$ related that the polarization switching time for PZT films using short voltage pulse technique was approximately $390 \times 10^{-12} \mathrm{~s}$. The switching data in FE were first analyzed by Ishibashi and Takagi ${ }^{[9]}$ in the framework of the Kolmogorov-Avrami (KA) theory. As we know, the KA theory is based on the nucleation and growth of domains of contradiction polarization. However, the KA theory being formulated for ideal systems frequently meets with obstacles while describing the kinetics of transformations in real objects characterized by violations of the two postulates: finiteness of transforming media and domain structure during polarization reversal in electric e.g in bulk single crystals, ceramics and thin films.

In the next portion, we will discuss the basic formalism on bulk FE switching in both transitions: first and second-order phase transition, respectively using Landau-Devonshire (LD) free-energy density and Landau-Khalatnikov (LK) equation of motion. The oscillatory switching formalism also implicates numerical approach where we use central finitedifference method (CFDM). Some basic discussion on switching based on free-energy and hysteresis loop are given in order to work out in detail the oscillatory switching behavior for bulk FE classifications.

\section{DIMENSIONLESS FORMALISM}

To deliberate the Landau model for switching, we may write all the formalism in dimensionless form to perform the universal results without any materialsparameter dependent using the conventional scaling $^{[1,3,6,10]}$ and the Landau free-energy expansion $\mathrm{f}$ for non-equilibrium behavior may be written as:

$$
f=\frac{1}{2} \alpha p^{2}-\beta p^{4}+\frac{1}{6} p^{6}-e p
$$

where for the first-order case, $\alpha=t_{r}$, while for secondorder, $\alpha=\left(t^{*}-1\right) . t_{r}$ and $t^{*}$ represents the dimensionless temperature for first and second-order phase transition, respectively. $\beta=1 / 2$ for first-order and $\beta=-1 / 4$ for the second-order case. The difference between these phase transitions is that first-order involves the expansion of $\mathrm{p}^{6}$, whereas $\mathrm{p}^{4}$ for second-order phase transitions. The last terms in (1) show the external electric field e as a driving force. Fig. 1 (a) and (b) show the free-energy $f$ curves switching due to the various electric field $\mathrm{e}$ (positive switching) for first and second-order phase transitions, respectively, the complete switching is affirmed by $\mathrm{e}=\mathrm{e}_{\mathrm{C}}$ (coercive field). The nonequilibrium free-energy curves are as pure-stable $\mathrm{FE}$ phase, where $t_{r}=-1$ (below supercooling temperature) and $\mathrm{t}=0.1$, for first and second-order case, respectively. As we know, in first-order conditions, there are various ranges of temperature ${ }^{[1,3]}$, but in this study we only focused on pure FE phase and it is enough for quantitative discussion. Differentiation of polarization due to the effect of we are given in both graphs, where A defined the bulk polarization $p_{B}$ value in an equilibrium state. The purple curves represent the complete switching from $\mathrm{e}=\mathrm{e}_{\mathrm{C}}$. Figure 1 (a) and (b) show the positive-side switching, with the bulk spontaneous polarization hello shifting the value of $\mathrm{A}$ to $\mathrm{p}_{\mathrm{B}}=-1.16$ and $\mathrm{p}_{\mathrm{B}}=-0.55$ for first and second-order phase transition respectively and vice versa for negative-side switching.

Coercive field: In FE materials, the polarization response to the electric field is highly non-linear and discloses a hysteresis loop. Electric field that has been applied may increase the ferroelectric domain until total domain growth and reorientation of all the domains have occurred in a direction favorable to the external field. At this juncture, the FE is tiveness deduced to acquire saturated polarization $\mathrm{p}_{\text {sat }}$ and if the electric field is abolished, some of the domains do not reorient into random configuration and, thus, the materials still polarized, so called remanent polarization, $\mathrm{p}_{\mathrm{r}}$. Then, the effect of electric field required to reestablish the polarization into zero called coercive field $\mathrm{e}_{\mathrm{c}}$. As mentioned above, e $>e_{c}$ is the inherent condition for spontaneous polarization to repeal their state.

Figure 2 (a) and (b) accords the dielectric hysteresis loops (DHL) curves (p versus e) at anomalous ranges of temperature at pure $\mathrm{FE}$ phase described by figure captions for first and second-order phase transitions, respectively, with the coercive field $\mathrm{e}_{\mathrm{c}}$ asserted by vertical arrows. The features in Fig. 2 (a) and (b) directly comes from $\mathrm{df} / \mathrm{dp}=0$ of (1) called the dielectric equation of state, given by:

$$
\frac{d f}{d p}=e=\alpha p-4 \beta p^{3}+p^{5}
$$

On the other hand, the DHL curves in Fig. 2 (a) and $2 \mathrm{~b}$ ) also describes the thermodynamically stable and unstable state, which is de/dp $>0$ and de/dp $<0$, respectively. The coercive field $\mathrm{e}_{\mathrm{c}}$ may be calculated directly from $\mathrm{d}^{2} \mathrm{f} / \mathrm{dp}^{2}=0$ of (1), since $\mathrm{e}_{\mathrm{c}}$ may be allocated at $\mathrm{p}$-e curves as inflection points, therefore the quadratic equation in $\mathrm{p}^{2}$ with solutions:

$$
\mathrm{p}_{\mathrm{C}}^{2}=\frac{3}{5}\left(1 \pm \sqrt{1-\frac{5}{9} \mathrm{t}_{\mathrm{r}}}\right) \text { and } \mathrm{p}_{\mathrm{C}}^{2}=\frac{\left(1-\mathrm{t}^{*}\right)}{3}
$$

For first and second-order case, respectively which is stated in Fig. 2 (a) and (b) in vertical axes. Substituting in (2) gives: 
$\mathrm{e}_{\mathrm{C}}=\frac{4}{5}\left[\frac{3}{5}\left(1 \pm \sqrt{1-\frac{5}{9} \mathrm{t}_{\mathrm{r}}}\right)\right]^{1 / 2}\left[\mathrm{t}_{\mathrm{r}}-\frac{3}{5}\left(1 \pm \sqrt{1-\frac{5}{9} \mathrm{t}_{\mathrm{r}}}\right)\right]$

And:

$$
e_{C}= \pm 2 \sqrt{\frac{\left(1-t^{*}\right)}{3}}
$$

the coercive field for first and second-order, respectively. The coercive field decreases correspond to the increase in temperature. This is clearly shown that the switching properties are highly affected by the temperature effect on high-temperature ranges-fieldinduced phase towards PE phase for first-order and near PE phase for second -order case.

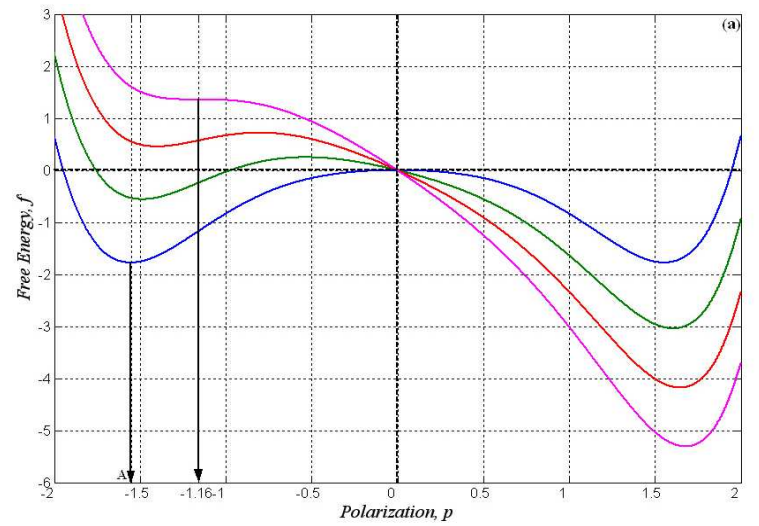

(a)

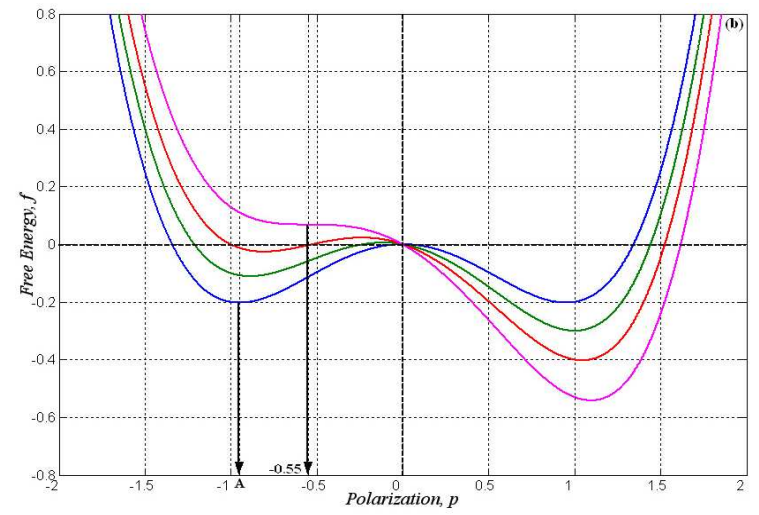

(b)

Fig. 1: Ferroelectric free energy $f$ versus polarization $p$ for (a) first-order and (b) second-order phase transition with temperature $t_{\mathrm{r}}=-1$ and $\mathrm{t}^{*}=0.1$, respectively. The curves represent positive switching calculated from different positive electric field: (a) for first and second-order case $\mathrm{e}=0$, equilibrium state $(\longrightarrow), \mathrm{e}_{1}=0.8, \mathrm{e}_{2}=0.1$ $(-), \mathrm{e}_{1}=1.5, \mathrm{e}_{2}=0.2(\Longrightarrow)$ and $\mathrm{e}_{1}=\mathrm{e}_{\mathrm{C} 1}=2.18$, $\mathrm{e}_{2}=\mathrm{e}_{\mathrm{c}}=0.33(\Longrightarrow)$. The polarization in an equilibrium state labeled with $\mathrm{A}$, where (a) $\mathrm{p}_{\mathrm{B}}=$ 1.55 and (b) $p_{B}=0.95$ for first and second-order case, respectively
Both values are indicated by vertical arrows in Fig. 2 (a) and 2 (b) for various temperatures and may be used as a guardian to determine the switching behavior, since e $\geq$ $e_{c}$ are the foremost conditions for the complete switching.

Oscillatory dynamic system switching: The atoms resonate proportionate to the effect of external electric field may described by Landau-Khalatnikov (LK) equation of motion as an oscillatory dynamical damped system, hence:

$\frac{\mathrm{d}^{2} \mathrm{p}}{\mathrm{d} \tau^{2}}+\mathrm{g}\left(\frac{\mathrm{dp}}{\mathrm{d} \tau}\right)=-\left(\frac{\delta \mathrm{f}}{\delta \mathrm{p}}\right)$

Which is evaluated from a spring oscillatory damped system where restoring force is eliminated.

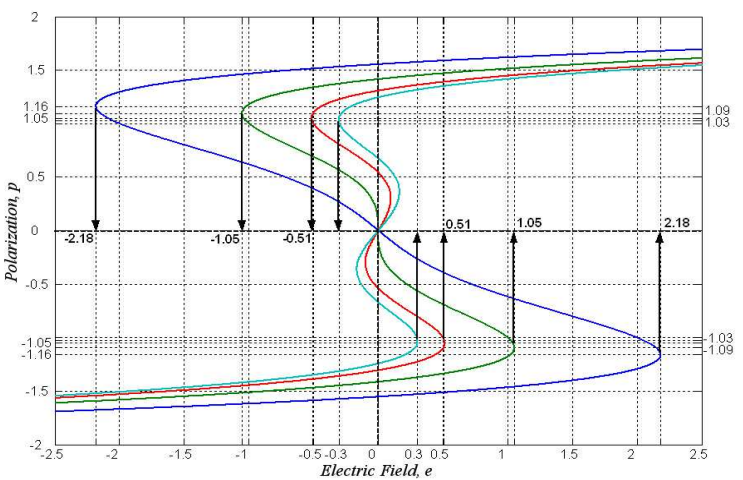

(a)

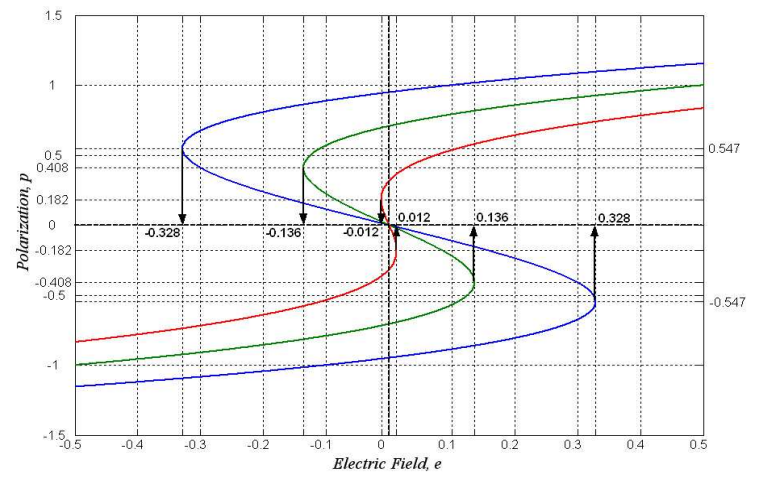

(b)

Fig. 2: Hysteresis loop p versus polarization e for (a) first-order and (b) second-order phase transition with different temperature at pure ferroelectric phase. For first-order case: $\mathrm{t}_{\mathrm{r}}=-1\left(\mathrm{t}<\mathrm{t}_{\mathrm{SHB}}\right)(-)$, $\operatorname{tr}=\mathrm{t}_{\mathrm{SH}}=0(\Longrightarrow), \mathrm{t}_{\mathrm{r}}=0.5(\Longrightarrow)$ and $\mathrm{t}_{\mathrm{r}} \approx \mathrm{t}_{\mathrm{CB}}=0.7$ (-) and for second-order case: $\mathrm{t}^{*}=0.1(-)$,

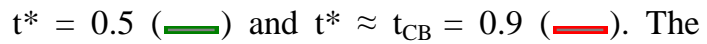
coercive field $e_{c}$ and polarization $p_{c}$ are labeled in both figures 




(a)

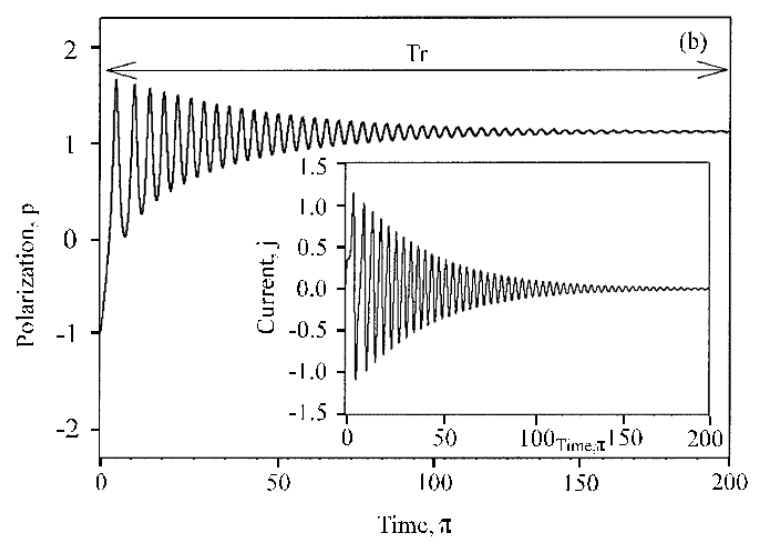

(b)

Fig. 3:Polarization $\mathrm{p}$ versus time $\mathrm{t}$ switching behaviors in (a) first-order and (b) second-order case with temperature $t_{r}=-1$ with $e=2.2$ (coercive electric field $\mathrm{e}_{\mathrm{c} 1}=2.18$ and damping parameter $\mathrm{g}=0.1$ ) and $\mathrm{t}^{*}=0.1$ with $\mathrm{e}=0.35$ (coercive electric field $\mathrm{e}_{\mathrm{c}}=0.33$ and damping parameter $\mathrm{g}=0.01$ ), respectively. The current, $\mathrm{j}$, switching in the inset for both cases

The second and first-order differential equation in the LHS of (6) describe the soft-mode dynamic properties in damped oscillatory system observed in manydisplacive FE. $g$ refer to the damping parameter:

$\mathrm{g}=\gamma\left(4 \varepsilon_{0} \mathrm{C} / \mathrm{B}^{2} \mathrm{~m}\right)^{1 / 2}$ and $\mathrm{g}=\gamma\left(\mathrm{a}^{3} \mathrm{~T}_{0}^{3} / \mathrm{m}_{0}{ }^{3}\right)^{1 / 2}$

which is for first and second-order phase transitions respectively. By applying (1) into (2), then:

$$
\frac{d^{2} p}{d \tau^{2}}+g \frac{d p}{d \tau}=-\alpha p+4 \beta p^{3}-p^{5}+e
$$

In relaxation system, the problem may be solved analytically since the first term of (7) vanishes but for oscillatory dynamical case, the numerical method approach is needed since we considered the second derivative term in (7).

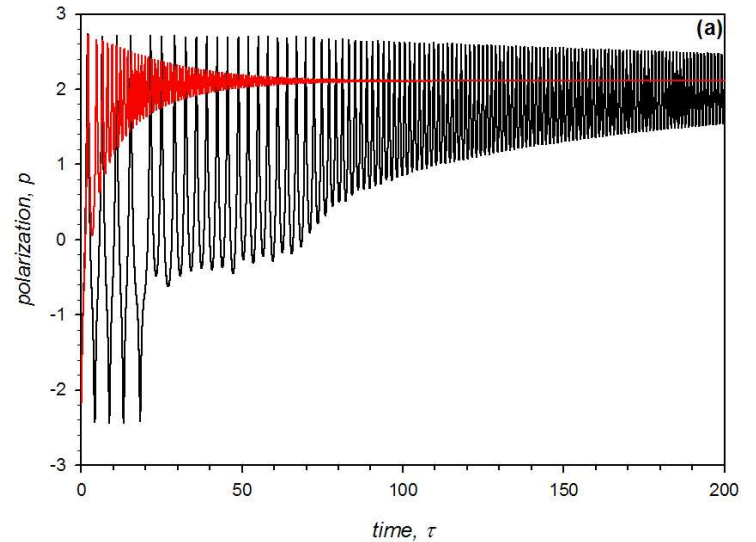

(a)

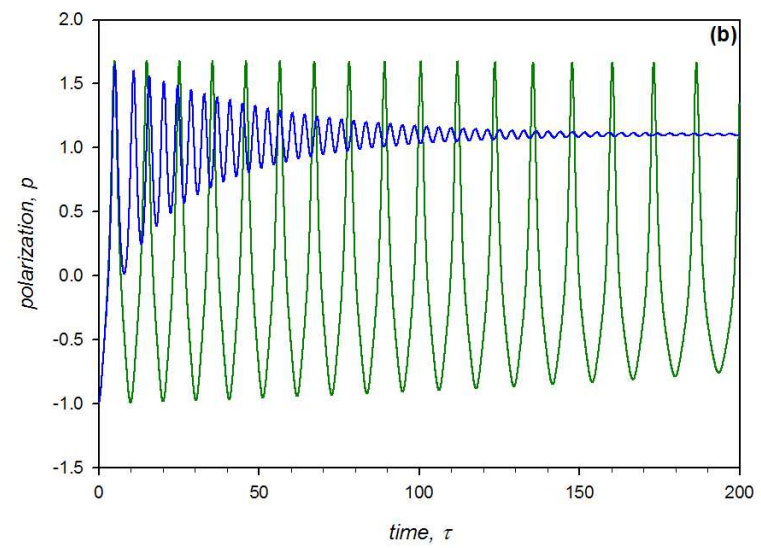

(b)

Fig. 4: Damping g effect for polarization switching: (a) first-order with $\mathrm{t}_{\mathrm{r}}=-1$ and $(\longrightarrow) \mathrm{g}=0.01,(\longrightarrow) \mathrm{g}$ $=0.1$ and $(\mathrm{b})$ second-order with $\mathrm{t}^{*}=0.1$ and $(\Longrightarrow)$ $\mathrm{g}=0.001,(\Longrightarrow) \mathrm{g}=0.05$. The electric field of curve (a) and (b) are the same as in Fig. 3

As described before $\mathrm{in}^{[6]}$, we are using Central Finite-Difference Approximation (CDFA) to treat the nonlinear equation of (7) and simultaneously may be written as:

$$
\mathrm{p}_{\mathrm{N}+1}=\frac{\begin{array}{l}
(\lambda-1) \mathrm{p}_{\mathrm{N}-1}+\left(2-\alpha(\Delta \tau)^{2}\right) \mathrm{p}_{\mathrm{N}} \\
+\left(4 \beta \mathrm{p}_{\mathrm{N}}^{3}-\mathrm{p}_{\mathrm{N}}^{5}+\mathrm{e}\right)(\Delta \tau)^{2}
\end{array}}{(1+\lambda))}
$$

where $\lambda=(1 / 2) g(\Delta \tau), \mathrm{p}_{\mathrm{N}+1}$ is the polarization to be computed, $\mathrm{p}_{\mathrm{N}-1}$ is the previous calculated value and $\mathrm{p}_{\mathrm{N}}$ the current value. The increment of time is given by $\Delta \tau$. Since the switching occurs in bulk FE, we take the initial polarization as $\mathrm{p}_{\mathrm{N}-1}=\mathrm{p}_{\mathrm{N}}=\mathrm{p}_{\mathrm{BULK}}$ for both cases, first and second-order, respectively. The current switching is defined as $\mathrm{j}=\left(\mathrm{p}_{\mathrm{N}+1}-\mathrm{p}_{\mathrm{N}-1}\right) /(2 \Delta \tau)$ using backward finite-difference approximation. Using some experimental data of $\mathrm{BaTiO}_{3}$ from Chew et al. ${ }^{[10]}$ and Wang et $a l .{ }^{[11]}$, hence the estimation of damping value for first and second-order are $\mathrm{g}=0.1$ and $\mathrm{g}=0.05$, 
respectively. The conditions for complete switching have been mentioned above where they correspond to the coercive field $e_{c}$ value and we assume the initial polarization occurred in negative states.

Figure 3 shows the under-damped oscillatory switching system behavior for first and second-order phase transition in 3 (a) and 3 (b) respectively. Since our proposition is to investigate the bulk FE oscillatory switching phenomenon, thus the temperatures at pure stable-state FE are considered, where $t_{r}=-1$ and $t^{*}=0.1$ for first and second-order phase transitions respectively. Clearly identity is that the transient response $(T R)$ in first-order is two times faster than second-order case, where the steady-state $(S S)$ occurred at $\tau \approx 100$. The transient response $(T R)$ of the dynamical system refers to the behavior as it makes a transition from the initial condition to the final condition, while the steady-state $(S S)$ is the area where the entire transient has died out $^{[12]}$. The settling time which is the time to reach the $S S$, is $\tau \sim 100$ and $\tau \sim 200$ for first and second-order respectively. The presence of damping parameter $\mathrm{g}$ in terms of mechanical vibration point of view makes oscillator always comes to rest as indicated in Fig. 4 for both first and second-order phase transitions. These motions are also known as transient vibrations. From Fig. 3 and 4, the presence of damping in simple harmonic oscillator (SHO) describe mathematically by Aexp (- $\eta \omega t)$, where $\eta$ is known as damping ratio, $A$ is the amplitude, $\omega$ is frequency and it is time.

\section{RESULTS AND DISCUSSION}

The oscillatory dynamical system for bulk ferroelectric in the first and second-order phase transitions has been studied in terms of switching time. All the calculations have been given in dimensionless form to perform the universal results. Details of dielectric hysteresis loops (DHL) for first and secondorder behavior has been discussed by investigating the coercive field $e_{c}$. Since the purpose of this study is to investigate the switching time $\mathrm{t}$ for bulk FE, then from Fig. 3, we know that the approximation of switching time $\mathrm{t}=\tau \mathrm{t}_{0}$. From previous study ${ }^{[3,6]}, \mathrm{t}_{0}=\left(\mathrm{m}_{0} / \mathrm{aT}_{0}\right)^{1 / 2}$ and $\mathrm{t}_{0}=\left(4 \mathrm{~m} \varepsilon_{0} \mathrm{C} / \mathrm{B}^{2}\right)^{1 / 2}$ for second and first-order phase transitions respectively. By using experimental data from Wang et al. ${ }^{[11]}$, thus $\mathrm{t}_{0} \sim 5.32 \times 10^{-16}$ s for secondorder case and $t_{0} \sim 7.38 \times 10^{-15}$ for first-order case. As mentioned above, from Fig. 3, the transient response $(T R)$ in first-order is two times faster than second-order case, where the steady-state $(S S)$ occurred at $\tau \approx 100$. Thus, the approximation values for first and secondorder phase transitions switching time in oscillatory dynamical system are $\mathrm{t} \sim 7.38 \times 10^{-13} \mathrm{~s}$ and $\mathrm{t} \sim 1.064 \times$ $10^{-13} \mathrm{~s}$ respectively. These results show that the approximation of theoretical calculation for bulk FE oscillatory switching time are in the ranges of $\sim 10^{-13} \mathrm{~s}$. Our theoretical estimation is valid since we have neglected other effects i.e domain wall movement and surface effect which occur in the FE thin film. The bulk switching time estimation that we calculated here may be used as theoretical-guide-value to estimate the oscillations switching in symmetric and asymmetric FE films and we are currently pursuing to investigate this behavior and hope to publish the results soon.

\section{ACKNOWLEDGEMENT}

The author would like to thank Assoc. Prof. Dr Zul Azhar Zahid Jamal, Dean of Microelectronic Engineering KUKUM for his support and corporation, and Assoc. Prof. A. Nazri Abdullah from PKKK for checking this manuscript.

\section{REFERENCES}

1. Lines, M.E. and A.M. Glass, 2001. Principles and Applications of Ferroelectrics and Related Materials, Clarendon Press, Oxford. New Edn.

2. Ricinschi, D., C. Harnagea, C. Papusoi, L. Mitoseriu, V. Tara and M. Okuyama, 1998. Analysis of ferroelectric switching in finite media as a Landau-type phase transitions. J. Phys. Condens. Matter, 10: 477.

3. Tan, E.K., Junaidah Osman and D.R. Tilley, 2001. Theory of switching in bulk first-order ferroelectric materials. Phys. Stat. Sol., 228: 765.

4. Merz, W.J., 1954. Domain Formation and Domain Wall Motions in Ferroelectric $\mathrm{BaTiO}_{3}$ Single Crystals. Phys. Rev., 95: 690.

5. Ya Shur, V., A.L. Gruverman, N. Yu Ponomarev, E.L. Rumyantsev, N.A. Tonkachyova, 1992. Integrated Ferroelectrics, 2: 51.

6. Halif, M.N.A. and Junaidah Osman, 2004. Analysis of bulk second-order ferroelectric switching in oscillatory dynamical system. KUKUM J. Eng. Res. and Education, 1: 97.

7. Larsen, P.K., G.L.M. Kampschoer, M.B. van der Mark and M. Klee, 1992. Proc. of the $8^{\text {th }}$ IEEE Intl. Symposium on Applications of Ferroelectrics (ISAF '92), pp: 217.

8. Larsen, P.K., R. Cuppens and G.J.M. Dormans, 1995. Science and Technology of Electronic Thin Films. Ed. By Auciello O and Waser R (Kluwer Academic, Dodrecht, The Netherlands), 284: 201.

9. Ishibashi, Y. and Y. Takagi, 1971. Note on ferroelectric domain switching. J. Phys. Soc. Jpn, 31: 506.

10. Chew, K-H., Junaidah Osman, R.L. Stamps, D.R. Tilley, J.F. Webb, 1999. Theory of far-infrared spectroscopy for ferroelectric size effect. Integrated Ferroelectrics, 23:161.

11. Wang, Y.G., W.L. Zhong and P.L. Zhang, 1994. Size effect on Curie temperature of ferroelectric particles. Solid State Commun., 92: 519.

12. Woods, R.L. and K.L. Lawrence, 1997. Modeling and Simulation of Dynamic Systems, Prentice Hall, New Jersey. 